\title{
Quaternion Hermitian Planes
}

\author{
Markus Stroppel
}

\begin{abstract}
The quaternion hermitian planes are defined, and are characterized by certain groups of automorphisms. For this purpose, characterizations of locally compact connected translation planes (in the context of stable planes) and compact connected projective desarguesian planes are given.
\end{abstract}

\section{Introduction.}

We study abelian groups of automorphisms of stable planes and characterize translation groups and their duals. This result is applied to stable planes of dimension 8 admitting unitary groups of degenerate hermitian forms on the three-dimensional left vector space over Hamilton's quaternions H. Together with previous results by R. Löwen [17] and the author [26: 7.8], this allows to determine the eight-dimensional point homogeneous stable planes admitting a unitary group $\Upsilon$ of $\mathrm{H}^{3}$ : any such plane is isomorphic with the subplane induced on some open orbit of $\Upsilon$ on the point set of the projective plane over Hamilton's quaternions, where the action of $\Upsilon$ is induced by the natural action of $\Upsilon$ on $\mathrm{H}^{3}$ or its dual. Partly, the results on stable planes seem to be new even in the more special context of (affine) locally compact connected translation planes.

We need a notion of topological dimension. For each stable plane, both the point and the line space are separable metric spaces [11: 1.9]. Consequently, their covering dimension coincides with any reasonable topological dimension function. We will denote the covering $\operatorname{dimension}$ of $A$ by $\operatorname{dim} A$. Note that the covering dimension of a real, complex or quaternion vector space coincides with its vector space dimension over the reals.

Endowed with the compact-open topology derived from the action on $M$, the group Aut (M) of all continuous collineations of a stable plane $\mathrm{M}=(M, \mathcal{M})$ becomes a locally compact transformation group on $M$ and $\mathcal{M}$ (see [11: 2.9]). A group of automorphisms of a stable plane is a topological group $\Delta$ together with a continuous monomorphism $\iota: \Delta \rightarrow$ Aut (M), where Aut (M) has the compact-open topology derived from the action

1991 Mathematics Subject Classification: 51H10, 51H20, 51A10, 51A45.

Key words and phrases: Stable planes, unitary groups, Hamilton's quaternions, locally compact connected translation planes, locally compact connected desarguesian planes. 
on $M$. For some results (especially criteria for compactness) it is necessary to make sure that $\iota$ is a homeomorphism onto $\Delta^{t}$. On the other hand there are some cases where one is interested in the possibilities for actions of abstract topological groups, e.g. if one deals with restrictions to invariant subplanes or with analytic subgroups of Lie groups. In the present paper we take the latter point of view. The groups that we are mainly interested in, however, are of such a kind that their toplogy is determined by the given (resp. the possible) actions. See [5: 5] for details. We shall consider locally compact groups only (recall that stabilizers, centralizers and normalizers are closed subgroups and therefore locally compact again, cf. [9: §26, Cor.3]).

\section{A characterization of the compact connected desarguesian projective planes.}

Recall that, up to isomorphism, there are precisely three compact connected desarguesian projective planes [22: Satz I]: namely the planes over the locally compact connected (skew) fields R (real numbers), C (complex numbers), and $\mathbf{H}$ (Hamilton's quaternions).

(2.1) Theorem. A compact connected projective translation plane $\mathbf{P}=(P, \mathcal{P})$ is desarguesian if, and only if, there is a locally compact group $\Phi$ of automorphisms of $\mathbf{P}$ such that the following hold for some $\mathrm{F} \in\{\mathbf{R}, \mathbf{C}, \mathrm{H}\}$ :

a) the group $\Phi$ fixes an affine point.

b) the group of translations is isomorphic with $\left(\mathrm{F}^{2},+\right)$ as a topological group, and, via conjugation in Aut $(P)$, the group $\Phi$ acts $F$-linearly on the group of translations.

c) If $\mathbf{F}=\mathbf{H}$ then the centralizer $\mathrm{C}_{\Phi}(\tau)$ has dimension $>1$ for each translation $\tau$. If $\mathrm{F}=\mathrm{C}$ then the centralizer $\mathrm{C}_{\boldsymbol{\Phi}}(\tau)$ contains more than two elements for each transla$\operatorname{tion} \tau$.

If $\mathbf{F}=\mathbf{R}$ then the centralizer $\mathrm{C}_{\Phi}(\tau)$ is non-trivial for each translation $\tau$.

Proof: i) If $\mathbf{P}$ is desarguesian, the stabilizer of any affine point contains a subgroup $\Phi \cong \mathrm{GL}_{2}(\mathrm{~F})$, and $\mathrm{C}_{\Phi}(\tau)$ contains a conjugate of the group $\left\{\left(\begin{array}{ll}1 & 0 \\ x & 1\end{array}\right) \mid x \in F\right\}$ of shears.

ii) We may identify the group $\mathrm{T}$ of translations with the set of affine points. Then $\Phi \leq$ $\mathrm{GL}_{2}$ (F). According to [1] (see [21: 8.1, Satz 2, p. 201], or [18: I.1, p. 1-7]), the plane $P$ is isomorphic with the desarguesian plane over $F$ if the stabilizer $\mathrm{T}_{L}$ of each line $L$ through $o=(0,0) \in F^{2}$ is an $F$-subspace of $T=F^{2}$. In the non-desarguesian case, one finds a translation $\tau \in \mathrm{F}^{2} \backslash\{(0,0)\}$ such that the $\mathrm{F}$-subspace $\Lambda$ spanned by $\tau$ is not contained in the line joining $o$ and $\tau$. The centralizer $C_{\Phi}(\tau)$ acts trivially on the point set $\Lambda$. If it is not contained in a line, then the set $\Lambda$ generates a Baer subplane or the whole plane, and assertion c) follows from [28: 5.1] and [28:6.3].

(2.2) Remark. A similar result holds without the assumption of connectedness for desarguesian planes over arbitrary locally compact (skew) fields: Assertion c) has to be replaced by the assertion that $\mathrm{C}_{\Phi}(\tau)$ is non-compact for each translation $\tau$, and the group $\Phi$ must be closed in Aut (P). 


\section{Abelian groups of automorphisms of stable planes.}

(3.1) Definitions.

a) A stable plane is a non-degenerate linear space $M=(M, \mathcal{M})$, where the point space $M$ and the line space $\mathcal{M}$ are endowed with locally compact Hausdorff topologies such that

- the covering dimension $\operatorname{dim} M$ is positive and finite

- joining of points and intersecting of lines are continuous operations

- the set of intersecting lines is open in $\mathcal{M} \times \mathcal{M}$.

b) For any subset $E \subseteq M$ that contains a quadrangle, a non-degenerate linear space $\mathrm{E}=(E, \mathcal{E})$ is induced on $E$, where $\mathcal{E} \subseteq \mathcal{M}$ consists of those lines that meet $E$ in more than one point. If $E \subseteq M$ is locally compact and $\operatorname{dim} E>0$, then $E$ is a stable plane. The linear space $E$ is called full in $M$ if any point that is incident with two lines of $\mathcal{E}$ belongs to $E$.

c) For any subset $X \subseteq M$ that contains a quadrangle, the subplane generated by $X$ in $\mathrm{M}$ is the smallest closed full subplane $\mathrm{E}=(E, \mathcal{E})$ of M satisfying $X \subseteq E$.

d) A stable plane is called almost projective if the complement of some line is an affine plane [13: 4.1].

(3.2) Remarks. General information about stable planes can be found in the work of R. Löwen ([11], [13], [15]). For our purposes, the following properties are of particular importance:

a) The only possible values for $\operatorname{dim} M$ are the integers $2,4,8$, and 16 (see [15: Th. 1]).

b) If $X$ is a connected subset of $M$ that is not contained in any line, then the subplane generated by $X$ is a stable plane.

c) For any proper closed full subplane $E=(E, \mathcal{E})$ of $\mathrm{M}=(M, \mathcal{M})$, we have that $\operatorname{dim} E<$ $\operatorname{dim} M$, see [28: 3.3].

d) For each point $p \in M$, the line pencil $\mathcal{M}_{p}=\{L \mid p \in L \in \mathcal{M}\}$ is a compact connected homotopy $l$-sphere, where $l=\operatorname{dim} \mathcal{M}_{p}=\operatorname{dim} L=\frac{1}{2} \operatorname{dim} M$ (see [11: 1.14, 1.17], [15: Th. 1, Th. 3]).

(3.3) Lemma. Let $\Delta$ be a connected locally compact abelian group of automorphisms of a stable plane $\mathrm{M}=(M, \mathcal{M})$ with $\operatorname{dim} M=2 l$. Then we have one of the following (mutually exclusive) cases:

a) $\Delta$ is quasi-perspective (i.e. each orbit in $M$ is contained in a line).

In this case, the stabilizer of any line moved by $\Delta$ is trivial, and $\operatorname{dim} \Delta \leq \operatorname{dim} M=2 l$.

b) There is a point $p \in M$ whose orbit $p^{\Delta}$ generates the whole plane.

Consequently, the stabilizer $\Delta_{p}$ is trivial, and $\operatorname{dim} \Delta \leq \operatorname{dim} M=2 l$.

c) There are points whose orbits are not contained in any line, but each of these orbits generates a proper subplane.

In this case, $\operatorname{dim} \Delta \leq \frac{3}{2} l<\operatorname{dim} M$.

Proof: Obviously, the three cases are mutually exclusive.

i) In case a), the orbit of each point $x$ moved by $\Delta$ is contained in a unique line $F_{x}$, which is fixed by $\Delta$. Consequently, the stabilizer $\Delta_{G}$ of a line $G$ moved by $\Delta$ acts trivially on $G$ and on each of the images of $G$. Having two axes, the group $\Delta_{G}$ is trivial. The bound for the dimension of $\Delta$ follows from [8]. 
ii) In case b), the stabilizer of $p$ acts trivially on $p^{\Delta}$ (since $\Delta$ is abelian), hence trivially on $M$ and is therefore trivial. The bound for the dimension of $\Delta$ follows from [8] again.

iii) In case c), we proceed by induction on $l$. For $l=1$, there are no proper full subplanes of $\mathrm{M}$, and case c) cannot occur. Assume that $l>1$. Then $l$ is one of the integers 2,4 , or 8 (see [15]). Let $\mathrm{E}=(E, \mathcal{E})$ be a proper $\Delta$-invariant subplane of maximal dimension. The locally compact connected abelian group $\Delta$ is a product $\Delta=\Phi \Theta$ of connected groups, where $\Phi$ acts almost effectively on $E$, while $\Theta$ is the connected component of the kernel of the action of $\Delta$ on $E$. By induction, we have that $\operatorname{dim} \Phi \leq \operatorname{dim} E \leq l$. Obviously, the assertion is true in the case where $\Theta=1$. If $\Phi=\mathbb{1}$, choose a line $L$ that contains at least two points of $E$. Then $L$ is fixed by $\Delta=\Theta$, and for any point $y \in L \backslash E$ we have that $\operatorname{dim} y^{\Delta} \leq l$. On the other hand, maximality of $\operatorname{dim} E$ yields that $\left\langle E, y^{\Delta}\right\rangle=\mathbf{M}$ and $\Delta_{y}=\mathbf{1}$. Thus we obtain that $\operatorname{dim} \Delta \leq l$. If $\Phi \neq \mathbf{1} \neq \Theta$, there is a line $L$ that contains at least two points of $E$ and is moved by $\Phi$. Choose $y \in L \backslash E$. Since $\left\langle E, y^{\Delta}\right\rangle=\mathbf{M}$, we have that $\Theta_{y}=1$ and $\operatorname{dim} \Theta=\operatorname{dim} y^{\Theta}$. The orbit $y^{\Delta}=y^{\Phi \Theta}$ generates a $\Delta$-invariant subplane of dimension $d$, and $d \geq 2 \operatorname{dim} y^{\Theta}=2 \operatorname{dim} \Theta$. By our assumption, we have that $d \leq \operatorname{dim} E \leq l$. Hence $\operatorname{dim} \Theta \leq \frac{l}{2}$, and the assertion follows from the fact that $\operatorname{dim} \Delta=\operatorname{dim} \bar{\Phi}+\operatorname{dim} \Theta$.

(3.4) Corollary. Let $\Delta$ be a connected locally compact abelian group of automorphisms of a stable plane $M=(M, M)$ and assume that $\operatorname{dim} \Delta=\operatorname{dim} M$. Then either $\Delta$ acts quasi-perspectively with an open orbit in $\mathcal{M}$, or $\Delta$ has an open orbit in $M$. In both cases, the action of $\Delta$ on the open orbit is sharply transitive.

(3.5) Examples.

a) On the projective quaternion plane, the 5-dimensional abelian group

$$
\left\{\left(\begin{array}{lll}
s & 0 & x \\
0 & s & y \\
0 & 0 & s
\end{array}\right) \mid s, x, y \in \mathrm{C}, s \bar{s}=1\right\}
$$

acts almost effectively and leaves invariant the complex subplane. Thus the bound in (3.3.c) is almost attained. Using results on the dimension of groups that act trivially on a subplane (cf. [12: 1.5], [26: 4.19] or [28: $5.1,7.6,8.21]$ ), this bound may be improved.

b) The bounds in (3.3.b), (3.3.a) are attained by full translation groups or their duals, respectively.

(3.6) Proposition.

Let $\mathrm{M}=(M, \mathcal{M})$ be a stable plane of dimension 2 , and let $\Delta \cong \mathbf{R}^{2 l}$ be a group of automorphisms of $\mathrm{M}$ such that each one-parameter subgroup acts quasi-perspectively.

a) If $\Delta$ is not quasi-perspective, then $\mathrm{M}$ is an almost projective translation plane with $\Delta$ acting as full group of translations.

b) If $\Delta$ is quasi-perspective, then the set $F$ of fixed points of $\Delta$ has at most one element. If such a point $z$ exists, each element $\delta \in \Delta$ has center $z$. For each point $x \in M \backslash F$, we have that $\Delta_{x} \cong \mathbf{R}^{l}$, and $\Delta_{x}$ acts sharply transitively on $\mathcal{M}_{x} \backslash\left\{F_{x}\right\}$, where $F_{x}$ is the line containing $x^{\Delta}$. In particular, the group $\Delta$ is transitive on $F_{x} \backslash F$, and $\Delta_{x}=\Delta_{\left[F_{x}\right]}$.

c) If $\Delta$ is quasi-perspective, then $M$ is a dual affine (or projective) translation plane if, and only if, the set $\mathcal{F}_{\Delta}=\left\{L \in \mathcal{M} \mid \exists x \in M:\{x\} \neq x^{\Delta} \subseteq L\right\}$ is compact.

d) We may identify $\Delta$ with the topological group $\left(\mathrm{F}^{2},+\right)$, where $F$ is the l-dimensional division algebra over $\mathbf{R}$ (i.e. $\mathrm{F} \in\{\mathbf{R}, \mathrm{C}, \mathrm{H}, \mathrm{O}\}$ ). If, with respect to such an identification, 
the stabilizer $\Delta_{x}$ is a line through the origin (i.e. an F-subspace in the cases $l \leq 4$ ) for each point $x \in M$, then the set $\left\{\Delta_{x} \mid x \in M \backslash F\right\}$ is an open subset of the pencil through the origin.

Proof: i) In case a), let $\Xi$ be a one-parameter group of $\Delta \cong \mathbf{R}^{2 l}$. Since $\Delta$ is abelian, the set $\mathcal{F}_{\Xi}=\left\{L \in \mathcal{M} \mid \exists x \in M:\{x\} \neq x^{\Xi} \subseteq L\right\}$ is left invariant by $\Delta$. By [12: 1.1] the projection $x \mapsto F_{x}$ (where $F_{x}$ is the line containing $x^{\Xi}$ ) induces a local homeomorphism of any line (moved by $\Delta$ ) to $\mathcal{F}_{\Xi}$. Thus $l=\operatorname{dim} \mathcal{F}_{\Xi} \geq \operatorname{dim} \Delta / \Delta_{G}$ for $G \in \mathcal{F}_{\Xi}$. On the other hand, $\Delta_{x}=1$ implies that $\operatorname{dim} \Delta_{G} \leq l$ and therefore $l=\operatorname{dim} \Delta_{G}=\operatorname{dim} \Delta / \Delta_{G}$. For any one-parameter group $\Theta$ of $\Delta$ not contained in $\Delta_{G}$, we consider the line $H$ that contains the orbit $x^{\ominus}$. Since $G \neq H$, we have that $\Delta_{G, H} \leq \Delta_{x}=\mathbf{1}$ and $\Delta_{G} \cong \mathbf{R}^{l} \cong \Delta_{H}$. According to [1], the orbit $x^{\Delta}$ carries an affine translation plane $A$. The stable plane $M$ contains $A$ and is contained in the projective hull of $A$, hence it is almost projective.

ii) In the quasi-perspective case, choose any line $L \neq F_{x}$ through a point $x$ that is not fixed by $\Delta$. Then $\Delta_{L}=1$, and $\operatorname{dim} \Delta / \Delta_{x} \leq l$. From the fact that $\operatorname{dim} \Delta_{x} / \Delta_{L} \leq l$ we deduce that $\operatorname{dim} \Delta_{x}=l=\operatorname{dim} L^{\Delta_{x}}$. Thus $L^{\Delta_{x}}$ is open in $\mathcal{M}_{x}$ for each $L \in \mathcal{M}_{x} \backslash\left\{F_{x}\right\}$. Since $\mathcal{M}_{x} \backslash\left\{F_{x}\right\}$ is connected, we infer that $\Delta_{x}$ acts sharply transitively on $\mathcal{M}_{x} \backslash\left\{F_{x}\right\}$, from which we obtain that $\mathcal{M}_{x} \backslash\left\{F_{x}\right\}$ is homeomorphic with $\mathbf{R}^{l}$ and $\Delta_{x} \cong \mathbf{R}^{l}$. Choose any point $y \in M \backslash F_{x}$ that is moved by $\Delta$. Then the line $x y \in \mathcal{M}_{x} \backslash\left\{F_{x}\right\}$ can be moved to $L$ by an element of $\Delta_{x}$, leaving invariant $F_{y}$. Therefore, each line $L \in \mathcal{M}_{x} \backslash\left\{F_{x}\right\}$ intersects with any line $F_{y} \in \mathcal{F}_{\Delta}$. Now $\Delta_{x}$ acts transitively on $F_{y} \backslash F$, and $\Delta_{y}$ fixes each point in $F_{y}$. iii) By ii), each line $L$ that is moved by $\Delta$ intersects with each line $F_{y} \in \mathcal{F}_{\Delta}$. If the set $\mathcal{F}_{\Delta}$ is compact, we conclude that $L$ is a compact line. Passing to the opposite plane [13: 1.1], case c) is reduced to case a). If $\Delta$ acts as a dual translation group, then $\mathcal{F}_{\Delta}$ equals the pencil through the center $z$ (cf. b)) and is therefore compact.

iv) Let $\mathcal{P}_{1} F$ denote the set of lines through the origin, endowed with the quotient topology from the mapping

$$
\pi: \Delta \backslash \mathbb{1}=F^{2} \backslash\{0\} \rightarrow \mathcal{P}_{1} F: \delta \mapsto F \delta
$$

Assume that $\Delta_{x} \in \mathcal{P}_{1} F$ for each point $x$. We choose a line $L$ that is moved by $\Delta$. According to ii), this line intersects with each line $F_{x} \in \mathcal{F}_{\Delta}$, and $\Delta_{x}=\Delta_{\left[F_{x}\right]}$. Now the mapping

$$
\mu: L \rightarrow \mathcal{P}_{1} \mathrm{~F}: x \mapsto \Delta_{x}
$$

is well defined and injective. For $x_{n} \in L$ we may choose $\delta_{n}$ in the unit sphere of $\Delta=\mathbf{R}^{2 l}$ such that $\Delta_{x_{n}}$ is the line joining the origin and $\delta_{n}$. Since the unit sphere is compact, there is a sequence $\delta_{n_{k}}$ converging to some $\delta$. For $x_{n} \rightarrow x$ we obtain that $\delta \in \Delta_{x}$. Thus $\Delta_{x}$ equals the line joining the origin and $\delta_{n}$, and the graph $\left\{\left(x, \Delta_{x}\right) \mid x \in L\right\}$ of $\mu$ is closed in $L \times \mathcal{P}_{1} \mathrm{~F}$. Since $\mathcal{P}_{1} \mathrm{~F}$ is compact, we conclude that $\mu$ is continous [3: XI, Th. 2.7]. Now the restriction of $\mu$ to any compact neighbourhood $C \subset L$ is a homeomorphism onto $C^{\mu}$. Since $\mathcal{P}_{1} F$ has the domain invariance property, we find that $\mu$ is an open embedding.

(3.7) Remarks.

a) In its natural (linear) action on the real affine plane, the group $\left\{\left({ }^{a}{ }_{b}\right) \mid a, b>0\right\} \cong \mathbf{R}^{2}$ is not a translation group and not quasi-perspective (indeed, the one-parameter 
subgroup $\left\{\left({ }^{a}{ }_{a^{-1}}\right) \mid a>0\right\}$ is not quasi-perspective). There are also compact 4 dimensional projective planes admitting a group of automorphisms that is isomorphic with $\mathbf{R}^{4}$ but is not a translation group, see [10: Satz 1,3)].

b) For $l=1$, there are no proper translation planes. Translation planes admitting large automorphism groups have been studied by $\mathrm{D}$. Betten in the case $l=2$. The cases $l=4,8$ have been treated by H. Hähl. See [2], [6], [7], and the references given there.

c) In the situation of $(3.6, d)$, there do occur proper open subsets of the pencil through the origin: E.g., let $\mathbf{P}$ be a compact connected projective translation plane with translation group $\Delta$. Then $\Delta$ acts quasi-perspectively on the dual $\mathbf{P}^{*}$ of $P$. Removing in $\mathbf{P}^{*}$ all the points that lie on lines in some closed set of lines through the common center of the elements of $\Delta$, we obtain a stable plane, on which $\Delta$ acts in the way described.

In many cases, one may check quasi-perspectivity of one-parameter groups by considering their centralizers in Aut (M). The following criterion, which is a generalization of (2.1), will be used later.

(3.8) Lemma. Let $\mathrm{F} \in\{\mathrm{R}, \mathrm{C}, \mathrm{H}\}$, and assume that a group $\Delta \cong \mathrm{F}^{2}$ of automorphisms of a stable plane $\mathrm{M}=(M, \mathcal{M})$ acts sharply transitively on $M$. Let $\Phi$ be a locally compact group of automorphisms of $M$ such that $\Phi$ fixes a point $p \in M$, normalizes $\Delta$ and acts $\mathrm{F}$-linearly on $\Delta=\mathrm{F}^{2}$ (via conjugation). If $\mathrm{F}=\mathrm{H}$ and $\operatorname{dim} \mathrm{C}_{\Phi}(\delta)>1$ for each $\delta \in \Delta$, or if $\mathbf{F} \in\{\mathbf{R}, \mathbf{C}\}$ and $\operatorname{dim} \mathrm{C}_{\Phi}(\delta) \geq 1$ for each $\delta \in \Delta$, then each one-parameter subgroup of $\Delta$ acts quasi-perspectively, and the geometry induced on $x^{\Delta}$ is the desarguesian affine plane with $\Delta$ acting as full group of translations.

Proof: Assume that there is a one-parameter subgroup $\Xi$ of $\Delta$ that does not act quasiperspectively. The $F$-subspace $\Lambda$ spanned by $\Xi$ is spanned by any element $\xi \in \Xi \backslash \mathbb{1}$. Since $\Delta$ acts transitively on $M$, the orbit $p^{\Xi}$ is not contained in a line. From the fact that $\Delta_{p}=1$ we infer that the subplane $E$ generated by $p^{\Lambda}$ is either a Baer subplane or equal to $M$. The group $\mathrm{C}_{\Phi}(\xi)$ acts trivially on $\Lambda$, hence trivially on $\mathrm{E}$, and we have reached a contradiction to $[28: 5.1,6.3]$ (cf. [28: 3.2]). The assertion follows from (2.1).

\section{Hermitian and dual hermitian quaternion planes.}

For any matrix $A=\left(a_{i j}\right)_{1 \leq i \leq n, 1 \leq j \leq m} \in \mathrm{H}^{n \times m}$, let

$$
{\overline{\left(a_{i j}\right)_{1} \leq i \leq n, 1 \leq j \leq m}}=\left(\bar{a}_{j i}\right)_{1 \leq j \leq m, 1 \leq i \leq n}
$$

denote the matrix obtained by conjugation of the entries and transposition. Observe that for each $y \in H^{3}$ we obtain that

$$
\bar{y}=\overline{\left(y_{1}, y_{2}, y_{3}\right)}=\left(\begin{array}{l}
\bar{y}_{1} \\
\bar{y}_{2} \\
\bar{y}_{3}
\end{array}\right) \text {. }
$$

(4.1) Definition. Let $f$ be one of the (skew) hermitian forms on $\mathbf{H}^{3}$ defined by $f(x, y)=$ $x J \bar{y}$, where $J$ is the diagonal matrix $\operatorname{diag}\left(v_{1}, v_{2}, v_{3}\right)$ with

$$
v=\left(v_{1}, v_{2}, v_{3}\right) \in\left\{\begin{array}{llll}
(1,1,1), & (1,1,-1), & (-1,-1,1), & (i, i, i) \\
(1,1,0), & (1,-1,0), & (-1,1,0), & (i, i, 0) \\
(1,0,0), & & (i, 0,0)
\end{array}\right\}
$$


In the hermitian cases (i.e. $v \in \mathbf{R}^{3}$ ), define

$$
M_{\left(v_{1}, v_{2}, v_{3}\right)}=\left\{H x \mid x \in H^{3} \backslash\{0\}, \quad x\left(\begin{array}{lll}
v_{1} & & \\
& v_{2} & \\
& & v_{3}
\end{array}\right) \bar{x}>0\right\} .
$$

In the skew hermitian cases, define

$$
M_{\left(v_{1}, v_{2}, v_{3}\right)}=\left\{H x \mid x \in \mathrm{H}^{3} \backslash\{0\}, \quad x\left(\begin{array}{ccc}
v_{1} & & \\
& v_{2} & \\
& & v_{3}
\end{array}\right) \bar{x} \neq 0\right\} .
$$

Then the sets $M_{v}$ are open, nonvoid subsets of the projective quaternion plane that are left invariant by the corresponding unitary group

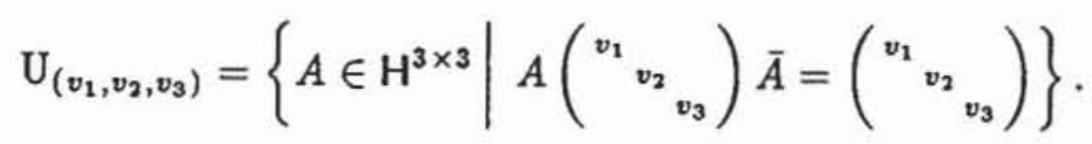

Moreover, this group acts transitively on the corresponding set $M_{v}$ by Witt's theorem [20]. For each $v$, the geometry $\mathrm{M}_{v}$ induced on $M_{v}$ (or $\mathrm{M}_{v}^{u}$ on $M_{v} \cup M_{-v}$, if $v \in \mathbf{R}^{3}$ ) is a stable plane with $\mathrm{U}_{v}$ acting almost effectively as a group of automorphisms. In accordance with R. Löwen [14], we call these planes the quaternion hermitian planes. The group $\mathrm{U}_{v}$ will be referred to as the induced group.

For convenience, we list the possibilities in a table. Let

$$
N_{\left(v_{1}, v_{2}, v_{3}\right)}=\left\{\mathrm{H} x \mid x \in \mathrm{H}^{3} \backslash\{0\}, \quad x\left(\begin{array}{lll}
v_{1} & & \\
& v_{2} & \\
& & v_{3}
\end{array}\right) \bar{x}=0\right\}
$$

denote the set of isotropic points. The point set $\{q \mid q \in L \in \mathcal{S}\}$ will be called a quaternion cylinder $\mathcal{C}_{k}$ if $\mathcal{S}$ is a subset of a line pencil $\mathcal{M}_{p}$ in the projective quaternion plane such that $\mathcal{S}$ is homeomorphic with the sphere $\mathbf{S}_{\boldsymbol{k}}$.

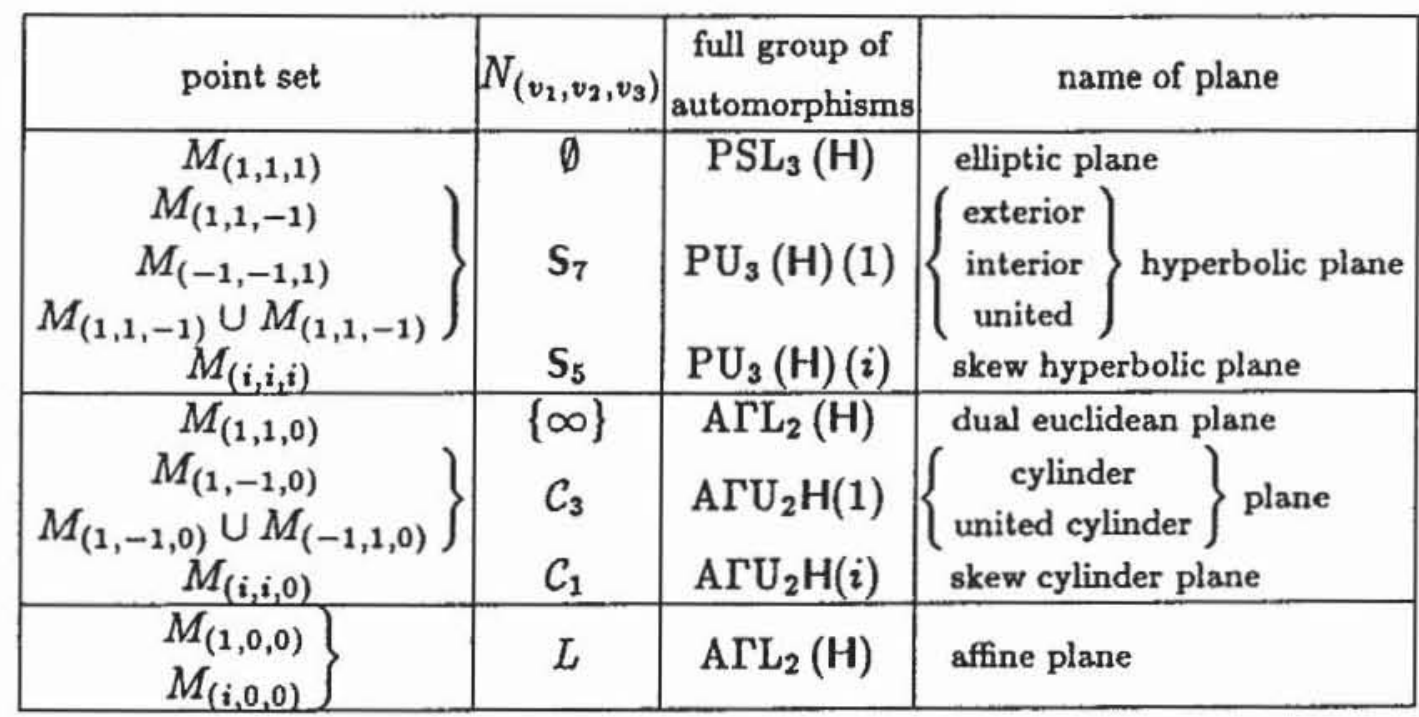


Note the curiosity that the familiar euclidean plane does not occur in this context (of course the underlying affine plane does occur, but the euclidean motion group only occurs in its dual action). The induced group differs from the full group of automorphisms in the cases where $v \in\{(1,1,1),(1,1,0),(i, 0,0)\}$.

Duality of the projective quaternion plane allows to introduce dual quaternion hermitian planes as follows: for any quaternion hermitian plane $\mathrm{M}_{v}=\left(M_{v}, \mathcal{M}\right)$ define the dual quaternion hermitian plane $\mathbf{D}=(D, \mathcal{D})$ to be the geometry induced on $D=\mathcal{M}$, considered as set of points in the dual projective plane. In the nondegenerate cases, we obtain the projective or the exterior hyperbolic plane. If $v \in\{(1,0,0),(i, 0,0)\}$, the dual plane is just the dual euclidean plane (a larger group is induced, however). For $v=(1,1,0)$, we obtain the euclidean (affine) plane. In the cases $v \in\{(1,-1,0),(i, i, 0)\}$ we get almost projective planes, and the induced groups contain the full translation group. There is exactly one open point orbit under the induced group in these cases, namely the affine plane. To these homogeneous open subplanes we refer as quaternion hermitian translation planes. Note that for these geometries the groups differ rather than the underlying affine planes.

(4.2) Remarks.

a) The hermitian planes induced on $M_{v}$, where

$$
v \in\left\{\begin{array}{ccc}
(1,1,1), & (1,1,-1), & (-1,-1,1), \quad(i, i, i) \\
(1,1,0), & (1,0,0), & (i, 0,0)
\end{array}\right\},
$$

are determined by the induced groups $\mathrm{U}_{v}$ (cf. [17], [26: 7.8]). For the cases $v \in$ $\{(1,1,0),(1,0,0),(i, 0,0)\}$, we give an independent proof (in terms of translation planes). In fact, we are going to characterize the quaternion hermitian planes and the quaternion hermitian translation planes by (subgroups of) the induced groups.

b) For stable planes of low dimension (i.e. 2 or 4 ), R. Löwen has characterized the hermitian planes in terms of existence of many reflections [14]. A similar attempt should work for quaternion hermitian planes. This is, however, beyond the scope of this note.

(4.3) Lemma.

Let $\Phi \Delta$ be a group of automorphisms of an 8-dimensional stable plane, with $\Delta \cong \mathbf{R}^{8}=\mathrm{H}^{2}$ and $\Phi=\mathrm{U}(J)$, where $J \in\left\{\left({ }_{1}{ }_{1}\right),\left({ }^{1}-1\right),\left({ }^{i} i_{i}\right)\right\}$ describes a non-degenerate (skew) hermitian form on $\mathrm{H}^{2}$. Assume that $\Phi$ acts on $\Delta$ in the usual, $\mathrm{H}$-linear way. If $\Delta$ acts quasi-perspectively, then the following hold:

a) There is a line $L$ such that $(\Phi \Delta)_{L}=\Phi$.

b) For each point $x \in L$, the stabilizer $\Phi_{x}$ equals the normalizer $N_{\Phi}\left(\Delta_{x}\right)$, and $(\Phi \Delta)_{x}=$ $\Phi_{x} \Delta_{x}$.

c) The central involution $\sigma=\left({ }^{-1}-1\right)$ of $\Phi$ has axis $L$.

d) The involution $\alpha=\left(\begin{array}{l}-1 \\ 1\end{array}\right) \in \Phi$ has at least one fixed point on $L$.

Proof: i) Choose any line $H$ that is moved by $\Delta$. According to (3.4), we have that $\Delta_{H}=1$. Let $\kappa: \Phi \Delta \rightarrow \Phi$ be the canonical mapping. We infer that $\left.\kappa\right|_{(\Phi \Delta)_{H}}$ is an isomorphism of $(\Phi \Delta)_{H}$ onto $\Phi$. Therefore $(\Phi \Delta)_{H}$ is a Levi-complement as well as $\Phi$, and there is some $\delta \in \Delta$ such that $\Phi=(\Phi \Delta)_{H}{ }^{\delta}=(\Phi \Delta)_{H^{\delta}}$. 
ii) From $\Delta_{x}=\Delta_{\left[F_{x}\right]}$ (cf. (3.6)) we obtain that the normalizer $N_{\Phi}\left(\Delta_{x}\right)$ fixes the axis $F_{x}$ and the intersection point $x=F_{x} \wedge L$. On the other hand, the stabilizer $\Phi_{x}$ fixes the line $F_{x}$ and therefore normalizes $\Delta_{\left[F_{x}\right]}$ (recall that $\Delta$ is normalized by $\Phi$ ). If $\varphi \notin \Phi_{x}$ then $x^{\varphi} \notin F_{x}$ and $x^{\varphi \Delta} \cap F_{x}=\emptyset$. Therefore $\varphi \Delta \cap(\Phi \Delta)_{x}=\emptyset$, and we have that $\varphi \in \Phi_{x}$ whenever $\varphi \delta \in(\Phi \Delta)_{x}$. We conclude that $(\Phi \Delta)_{x}=\Phi_{x} \Delta_{x}$. This proves assertion b).

iii) The involution $\sigma$ normalizes each R-subspace of $\Delta$. Therefore assertion b) yields that $\sigma \in \Phi_{[L]}$, and assertion c) is proved.

iv) For each $\delta \in \Delta_{x} \backslash \mathbf{1}$, the centralizer $\mathrm{C}_{\Phi}(\delta)$ fixes the axis $F_{x}$ of $\delta$ and is therefore contained in $\Phi_{x}$. For each non-isotropic $\delta$ (with respect to the sesquilinear form induced by $J$ on $\Delta$ ), there is a conjugate of $\alpha$ in $\mathrm{C}_{\Phi}(\delta)$. If $\alpha$ has no fixed points in $L$, we have

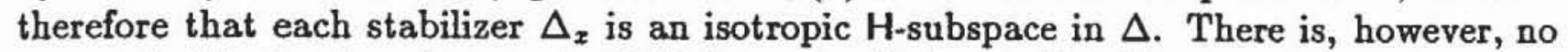
open set of isotropic subspaces in $\mathcal{P}_{1} \mathrm{H}$, in contradiction to (3.6.d).

(4.4) Proposition. Let $\Phi \Delta$ be a group of automorphisms of an eight-dimensional stable plane, with $\Delta \cong \mathrm{R}^{8}=\mathrm{H}^{2}$ and $\Phi=\mathrm{U}(J)$, where $J \in\left\{\left(\begin{array}{l}1 \\ 1_{1}\end{array}\right),\left(\begin{array}{c}1 \\ -1\end{array}\right),\left({ }^{i}{ }_{i}\right)\right\}$ describes a non-degenerate (skew) hermitian form on $\mathrm{H}^{2}$. Assume that $\Phi$ acts on $\Delta$ in the usual, H-linear way.

a) If $\Delta$ is not quasi-perspective, then $\mathrm{M}$ is isomorphic with the plane induced on the complement of some closed subset of a line in the projective quaternion plane, and the group $\Phi \Delta$ acts in the usual way.

b) If $\Delta$ is quasiperspective and $J=\left(l_{1}\right)$, then the stable plane $M$ is isomorphic with the dual euclidean quaternion plane, or its projective closure.

c) If $\Delta$ is quasi-perspective and $J=\left(\begin{array}{l}1 \\ -1\end{array}\right)$, then the stable plane $M$ has an open $\Phi \Delta$. invariant subplane that is isomorphic with the cylinder plane $\mathrm{M}_{(1,-1,0)}$.

d) If $\Delta$ is quasi-perspective, $J=\left({ }^{i}{ }_{i}\right)$, and the involution $\alpha=\left(\begin{array}{l}-1 \\ 1\end{array}\right)$ is not planar, then the stable plane $\mathrm{M}$ has an open $\Phi \Delta$-invariant subplane that is isomorphic with the skew cylinder plane $\mathbf{M}_{(i, i, 0)}$.

Proof: i) Assume that $\Delta$ is not quasi-perspective. According to (3.4), there is a point $p$ such that $\Delta_{p}=1$. Comparing dimensions, we obtain that $(\Phi \Delta)_{p}=(\Phi \Delta)_{p} / \Delta_{p} \cong \Phi$. Since $\Phi$ is a Levi-complement, we may assume that $\Phi=(\Phi \Delta)_{p}$. Using Witt's theorem [20], the centralizer $\mathrm{C}_{\Phi}(\delta)$ is computed easily for each $\delta \in \Delta$. Now assertion a) follows from (3.8). ii) Assume that $\Delta$ acts quasi-perspectively, and $J=\left({ }_{1}{ }_{1}\right)$. Then the compact group $\Phi=$ $\mathrm{U}_{2}(\mathrm{H})$ acts transitively on the connected set $\mathcal{F}_{\Delta}=\left\{L \in \mathcal{M} \mid \exists x \in M:\{x\} \neq x^{\Delta} \subseteq L\right\}$ (cf. [19: Th. 1]). By (3.6.b), the plane $\mathrm{M}$ is a dual affine translation plane or its projective closure. Passing to the dual plane reduces this situation to case a).

iii) Assume that $J=\left({ }^{1}-1\right)$. According to (4.3) and [26:4.19] or [28: 6.5], the involution $\alpha$ is neither free nor planar. Therefore there is a point $x \in L$ that is the center or lies on the axis of $\alpha$. In the first case, we obtain that $\Delta_{x}=\mathrm{C}_{\Delta}(\alpha)$, and $\Phi_{x}=\mathrm{N}_{\Phi}\left(\mathrm{C}_{\Delta}(\alpha)\right.$ ) by (4.3.b). In the second case, we have that $\alpha \sigma$ fixes the axes of $\alpha$ and $\sigma$. Since these axes intersect in $x$, and since commuting involutions cannot have the same axis [29: 9], we obtain that $\alpha \sigma \in \Phi_{[x]}$. Now $\left({ }_{1}^{1}\right)$ induces an (outer) automorphism of $\Phi$, interchanging $\alpha$ and $\alpha \sigma$. Therefore the stabilizer $(\Phi \Delta)_{x}$ is determined. According to (3.6.b), there are two line 
orbits in $\mathcal{M}_{x}$, namely $\left\{F_{x}\right\}$ and $\mathcal{M}_{x} \backslash\left\{F_{x}\right\}=L^{\Delta_{x}}$. Now $(\Phi \Delta)_{L}=\Phi$ and $(\Phi \Delta)_{F_{x}}=\Phi_{x} \Delta$, and the geometry induced on $x^{\Phi \Delta}$ can be reconstructed according to [27] or [26:6.3]. iv) Assume that $J=\left({ }_{i}{ }_{i}\right)$. According to (4.3), the involution $\alpha$ is not free. By assumption, $\alpha$ is not planar, and we can proceed analogously to iii) (recall that $\alpha$ and $\alpha \sigma$ are conjugate in $\mathrm{U}(J))$.

(4.5) Remark. The groups considered in (4.4) are proper subgroups of $U_{(1,1,0)}, U_{(1,-1,0)}$ and $\mathrm{U}_{(i, i, 0)}$, respectively. For the skew cylinder planes the full group $\mathrm{U}_{(i, i, 0)}$ yields that $\alpha$ is not planar: the centralizer of $\alpha$ in $U_{(i, i, 0)}$ contains the group $\Sigma T \Xi$, where

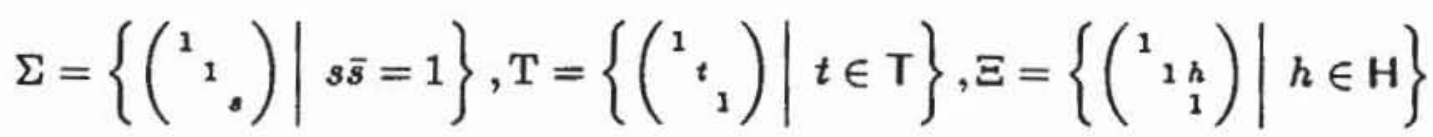

According to [17], this group cannot act almost effectively on a four-dimensional plane. Since $\Sigma T \Xi$ has no normal subgroup of dimension 1, we obtain from [26:4.19] or [28:6.3] that $\alpha$ is not planar.

Restricting ourselves to point homogeneous stable planes, we can state the following result: (4.6) Theorem. Among the point homogeneous stable planes of dimension 8 , the quaternion hermitian planes are, up to duality and isomorphism, determined uniquely by the corresponding unitary groups.

Proof: Let $\mathrm{M}_{v}$ be a quaternion hermitian plane with induced group $\mathrm{U}_{v}$. If $v \in$ $\{(1,1,1),(1,-1,-1),(-1,-1,1)\}$ the assertion has been proved by $\mathrm{R}$. Löwen [17]. Note that $\mathrm{M}_{(1,1,-1)}$ is the dual of $\mathrm{M}_{(-1,-1,1)}$. The case where $v=(i, i, i)$ has been treated by the author in [26: 7.8]. The remaining cases (where the hermitian form is degenerated and the induced group is not simple but of mixed type) are covered by Proposition (4.4).

(4.7) Remark. The assumption of point-homogeneity is necessary to make the reconstruction method of [27] applicable. There remains the problem to decide whether each stable plane that admits one of the groups $U_{v}$ is isomorphic with some $U_{v}$-invariant subplane of the projective plane over $\mathrm{H}$.

\section{References.}

1. André, J., 'Ūber nicht-Desarguessche Ebenen mit transitiver Translationsgruppe', Math. Z. 60 (1954) 156-186.

2. Betten, D., '4-dimensionale Translationsebenen mit kommutativer Standgruppe', Math. Z. 154 (1977) 121-141.

3. Dugundji, J., Topology, Allyn and Bacon, Boston, 1966.

4. Freudenthal, H., 'Einige Sātze über topologische Gruppen', Ann. of Math. 37 (1936) 46-56.

5. Grundhöfer, T., and Stroppel, M., 'On restrictions of groups of automorphisms of compact connected projective planes to subplanes', Res. Math. 21 (1992) 319-327.

6. Hähl, H., 'Achtdimensionale lokalkompakte Translationsebenen mit mindestens 17-dimensionaler Kollineationsgruppe', Geom. Ded. 21 (1986) 299-340.

7. Hähl, H., 'Die Oktavenebene als Translationsebene mit großer Kollineationsgruppe', Monatsh. Math. 106 (1988) 265-299.

8. Halder, H.R., 'Die Dimension der Bahnen lokalkompakter Gruppen', Arch. der Math. 22 (1971) 302-303.

9. Husain, T., Introduction to topological groups, Saunders, Philadelphia, London, 1966. 
10. Knarr, N., '4-dimensionale projektive Ebenen mit großer abelscher Kollineationsgruppe', J. Geom. 31 (1988) 114-124.

11. Löwen, R., 'Vierdimensionale stabile Ebenen', Geom. Ded. 5 (1976) 239-294.

12. Löwen, R., 'Halbeinfache Automorphismengruppen von vierdimensionalen stabilen Ebenen sind quasi-einfach', Math. Ann. 236 (1978) 15-28.

13. Löwen, R., 'Central collineations and the Parallel Axiom in stable planes', Geom. Ded. 10 (1981) 283-315. 14. Löwen, R., 'Stable planes of low dimension admitting reflections at many lines', Res. Math. 5 (1982)
$60-80$.

15. Löwen, R., 'Topology and dimension of stable planes: On a conjecture by H. Freudenthal', J. Reine Angev. Math. 343 (1983) 108-122.

16. Lōwen, R., 'Actions of $\mathrm{Spin}_{3}$ on 4-dimensional stable planes', Geom. Ded. 21 (1986) 1-12.

17. Löwen, R., 'Stable Planes admitting a Classical Motion Group', Res. Math. 8 (1986) 119-130.

18. Lüneburg, H., Thanslation planes, Springer, Berlin etc., 1980.

19. Mann, L.N., 'Gaps in the dimensions of compact transformation groups', Mlinois J. Math. 10 (1966) 532-546.

20. Pall, G., 'Hermitian quadratic forms in a quasi-field', Bull. Am. Math. Soc. 51 (1945) 889-893.

21. Pickert, G., Projektive Ebenen, Springer, Berlin etc., 1955.

22. Pontrjagin, L.S., 'Über stetige algebraische Körper', Ann. Math. 33 (1932) 163-174.

23. Salzmann, H., 'Topological Planes', Adv. in Math. 2 (1969) 1-60.

24. Salzmann, H., 'Compact 8-dimensional projective planes with large collineation groups', Geom. Ded. 8 (1979) 139-161.

25. Skornjakov, L.A., 'Topological projective planes', Trudy Moskov. Mat. Obšc. 3 (1954) 347-373.

26. Stroppel, M., Achtdimensionale stabile Ebenen mit quasieinfacher Automorphismengruppe, Dissertation, Tübingen, 1991.

27. Stroppel, M., 'Reconstruction of incidence geometries from groups of automorphisms', Archiv der Math. (1992) (to appear).

28. Stroppel, M., 'Planar groups of automorphisms of stable planes', J. Geom. (1992) (to appear).

29. Stroppel, M., Quasi-perspectivities in stable planes, Preprint No. 1462, Math. Institut der Technischen Hochschule Darmstadt, 1992.

Author's address:

Markus Stroppel

Fachbereich Mathematik

Technische Hochschule Darmstadt

SchloBgartenstr. 7

D-6100 Darmstadt

stroppel@mathematik.th-darmstadt.de

Eingegangen am 7. April 1992. 